\title{
Pharmacy students' knowledge on Human Papillomavirus, its implication in cervical cancer and the need for vaccination
}

Authors: Rosalind Wyatt, Cara Cully, Kay Marshall, Kaye Williams, Rebecca Elvey. Manchester Pharmacy School, The University of Manchester

Background: The United Kingdom (UK) has a National Human Papillomavirus (HPV) Vaccination Programme. To advise the public it is important that fourth year pharmacy students have sufficient knowledge on the HPV vaccine. The ultimate aim of this research was to develop a leaflet suitable for pharmacy students and pharmacists about the National HPV Vaccination Programme.

Method: The literature was reviewed to determine the basic science required by pharmacists to appropriately advise the public on the HPV vaccine, and to gain scientific knowledge in those areas. A questionnaire was produced to collect data from fourth year students attending one UK school of pharmacy. The data were analysed to assess the students' knowledge level on HPV. The leaflet was produced by considering areas of poor knowledge and the information required to advise.

Results: Questionnaires were distributed to 159 students and the response rate was $132 / 159$ (83\%). Overall knowledge was low, with a mean total score of $11.8 / 22$. Out of the 15 questions assessing participants' knowledge on cervical cancer, HPV and the HPV vaccine, Gardasil $\circledast$, 7 were 'well answered' (>60\% of participants answered correctly), 4 were 'moderately well answered' (40-60\% of participants answered correctly) and 4 were 'poorly answered' ( $<40 \%$ of participants answered correctly). Areas of low knowledge included prevalence and transmission of HPV, the recommended dosing schedule of Gardasil@ and whether it confers cross-protection.

Conclusions: This study highlights the lack of sufficient knowledge held by fourth year pharmacy students at the studied school of pharmacy to appropriately advise the public. The leaflet developed aims to inform students and pharmacists to increase their knowledge on HPV vaccination, but the effectiveness of this tool is unknown. Pharmacy students need to be better informed on the National HPV Vaccination Programme so that they can advise the public appropriately and increase uptake of the vaccine. 